Matrices (FR and NO), in approximately 316000 persons representing 3.6 million person-years of follow up. '

Results Results for Non-Hodgkin lymphomas (NHL) were recently published, based on 2430 cases. Most meta-HRs suggested no association. Moderately elevated meta-HRs were seen for NHL and ever use of terbufos (meta-HR=1.18, 95\% CI: 1.00-1.39), based on 300 exposed cases; chronic lymphocytic leukaemia/small lymphocytic lymphoma and deltamethrin (1.48, 1.06-2.07), 148 cases; and diffuse large B-cell lymphoma and glyphosate (1.36, 1.00-1.85), 221 cases; although the latter was driven exclusively by results in the Norwegian cohort. Inverse associations of NHL with the broader groups of organochlorine insecticides $(0.86,0.74-0.99)$ and phenoxy herbicides $(0.81,0.67-0.98)$ were observed, but individual active ingredients within these groups were not associated after adjusting for exposure to other pesticides. Preliminary results based on data from the US and NO for myeloid cancers indicate moderate associations with dichlorvos and aldicarb.

Conclusion Individual studies are often underpowered regarding their ability to demonstrate associations of disease, particularly for more rare exposures or for rare cancers or subtypes. Analyses within the AGRICOH consortium provide an opportunity to overcome the low power to detect.

\section{S-344 NEUROLOGICAL DISEASES IN FARMERS: OPPORTUNITIES WITHIN THE AGRICOH CONSORTIUM}

${ }^{1}$ Isabelle Baldi, Anne-Helen Harding, Christine G Parks, Sue Park, Joachim Schuz, Aesun Shin, Srishti Shrestha, Pierre Lebailly. 'University of Bordeaux, France

\subsection{6/OEM-2021-EPI.438}

Objectives In most countries, the burden of neurological disorders is shown to increase. One explanation is the increase in life expectancy, especially for neurodegenerative ones. Environmental factors, including chemicals such as pesticides may also explain this Public Health issue. Several epidemiological studies have shown pesticide exposure in farmers was associated with neurodegenerative diseases. The strongest evidence concerns Parkinson's disease, but there are also data for Alzheimer's disease and amyotrophic lateral sclerosis. In addition, several studies have suggested that farmers had higher depression and suicide rates than the general population, which could be explained by several factors like remote residence, long working days, time pressure, economic stressors. Pesticides are also suspected as some of them disturb neurotransmitters implicated in mood, like serotonin and dopamine.

Methods In AGRICOH, there are opportunities to further study the occurrence of neurological diseases in relation with agricultural exposures. A first step has been taken by describing mortality from neurodegenerative diseases and suicide in the 4 cohorts who collected this type of data: the Agricultural Health Study in US $(\mathrm{N}=51,502,1999-2015)$, the AGRICAN cohort in France $(\mathrm{N}=181,842,2005-2015)$, the Prospective Investigation of Pesticide Applicators' Health Study in UK ( $\mathrm{N}=4,944$ participants, 2013-2016) and the Korean Multicenter Cancer Cohort study ( $\mathrm{N}=8,428$ participants; 1993-2013). Standardized mortality ratios were calculated according to gender and countries.

Results In these first analyses, no excess in neurodegenerative mortality in males and females was observed in the four cohorts. However, suicide mortality was significantly increased in French, British and Korean females $(\mathrm{SMR}=1.46 ; 23 ; 1.66$, respectively) and in French males $(\mathrm{SMR}=1.13,1.03-1.25)$ compared to the general population.

Conclusion Possibilities to go further on neurological diseases in AGRICOH, exploring possibilities to get incidence data, to combine data from additional cohorts, to include metrics for pesticide exposures in the analysis will be discussed during this symposium.

\section{S-388 EXPOSURE ASSESSMENT FOR POOLING PROJECTS WITHIN THE AGRICOH CONSORTIUM}

${ }^{1}$ Hans Kromhout. ' Utrecht University, Netherlands

\subsection{6/OEM-2021-EPI.439}

Objectives Pooling epidemiological studies can be beneficial when studying rare outcomes and subtypes of diseases. However, pooling of exposure information is a challenge since standard approaches are not employed. The objective of this undertaking was to enable pooling of agricultural cohort studies at level of exposure to active ingredients.

Methods Initial efforts in AGRICOH focused on cancer outcomes in three large cohort studies, the Agricultural Health Study (AHS-USA), Agriculture and cancer (AGRICAN-France) and Cancer in the Norwegian Agricultural Population (CNAPNorway) with data from $>300000$ individuals. Each study employed different methods, including Crop-Exposure Matrices (CEM), (AGRICAN and CNAP), and self-report (AHS), which were compared.

Results The CEM approach led to much higher prevalence of exposures. The CEM approach as expected generated false positive exposures. Lack of specificity is less of an issue for pesticides applied relatively frequently, but will lead to more exposure misclassification when prevalence of use is low. Given that assignment of exposure will be independent of disease status, misclassification is non-differential and will result in bias towards the null, especially when strength of association is modest, as for most agricultural exposures and health effects. Within AGRICOH our ability to detect associations are for now at best limited without further improvement of exposure assessment and assignment.

Conclusion Clear differences in farming systems, crops and animals, climate and agricultural inputs do exist and may result in large qualitative and quantitative differences in exposure when accurately assessed and assigned. Consequently, it may provide opportunities to evaluate exposure contrasts.To enable pooling in more informative ways, further thought is given within AGRICOH to collect agricultural occupational histories in more detail and to harmonize exposure assessment and assignment. Standardization of exposure assessment approaches within AGRICOH and future studies is a necessity and will make pooling easier and may result in more informative studies.

\section{S-419 LONG TERM EFFECTS OF COVID-19 IN HEALTH WORKERS AND THE ASSESSMENT OF THE FATIGUE SYNDROME IN COMPENSATION CLAIMS}

${ }^{1}$ Albert Nienhaus. 'University Clinics Hamburg Eppendorf (UKE), Germany

10.1136/OEM-2021-EPI.440 
Introduction In Germany more than 45,000 cases of COVID19 are already recognized as Occupational Disease (OD) for workers in the health and welfare sector. Different long term effects of COVID-19 are described in literature, fatigue being one of the most common symptoms observed after the infection.

Objectives We assessed long term effects of COVID-19 three months and more after the SARS-CoV-2 infection in health and social workers (HSW).

Methods A self-administered questionnaire was sent to 3,800 HSW with COVID-19 recognized as OD in January 2021. The questionnaire was self-developed and assessed biographical data, symptoms at time of infection, quality of life (QoL) and workability as well as COVID-19 associated symptoms three months or more after infection. The study was approved by the Medical Ethic Committee in Hamburg, Germany.

Results A total of 2,018 HSW returned the questionnaire. Response rate was 53\%. $82 \%$ were female and 52\% were older than 50 years. $42 \%$ worked in hospitals and $30 \%$ in nursing homes, 9\% were doctors and 56\% nurses. Compared to the time before the infection, QoL was reduced by $72 \%$ and work ability was reduced by $83 \%$. Severe fatigue was reported by $20 \%$, sever concentration and memory problems by $14 \%$ and severe breathlessness by $9 \%$. 4.5\% had already taken part in a rehabilitation and $37 \%$ wished to take part in a rehabilitation.

Conclusion Long term fatigue symptoms are common in HSW more than three months after COVID-19. The need for rehabilitation in HSW with COVID-19 as OD is high. The further cause and the treatment options for long term effects and especially for fatigue need to be studied.

\section{S-452 CHALLENGES OF INTEGRATING GENDER THEORETICAL CONCEPTS INTO EPIDEMIOLOGICAL HEALTH RESEARCH}

${ }^{1}$ Gabriele Bolte. 'Institute of Public Health and Nursing Research, University of Bremen, Germany

\subsection{6/OEM-2021-EPI.441}

In recent years awareness of the need to integrate sex/gender more comprehensively into epidemiological health research has increased. However, a discrepancy between gender theoretical concepts on one hand and epidemiological practice on the other hand still remains. A conceptualization of a static, individual characteristic sex/gender (often only dichotomous as male/female) does not capture variability, multidimensionality and contextuality of sex/gender. Moreover, interactions between sex-linked biology and gender relations, processes of embodiment as well as intersectionality in terms of power relations and processes of privilege or discrimination are hardly considered. This is especially true for environmental health research.

The presentation will draw on experiences gathered in two ongoing research projects: INGER and AdvanceDataAnalysis. The collaborative research project INGER (Integrating gender into environmental health research) developed a multidimensional sex/gender concept from an intersectionality perspective. This concept guided operationalizations for sex/gender-related data collection in a population-based study. Decision tree methods are currently applied to assess the relevance of several sex/gender dimensions when identifying subgroups with especially high environmental exposures. The subproject
AdvanceDataAnalysis as part of the collaborative research project AdvanceGender focuses on the more in-depth analysis of already existing data to support gender-sensitive health reporting. One approach is to define 'solution-linked' variables that indicate modifiable societal and contextual factors and help to explain heterogeneity across social dimensions.

With INGER and AdvancDatAnalysis as examples, challenges in operationalization of sex/gender based on gender theoretical concepts, and in statistical analysis of the impact of multiple sex/gender dimensions on exposure variation and effect modification will be discussed.

Improvements in the integration of theoretically sound sex/ gender concepts in epidemiological health research will enhance its validity and significance and, in perspective, contribute to more health equity.

\section{S-464 AUTOMATED OCCUPATIONAL ENCODING TO THE CANADIAN NATIONAL OCCUPATION CLASSIFICATION USING AN ENSEMBLE CLASSIFIER FROM TF-IDF AND DOC2VEC EMBEDDINGS}

${ }^{1}$ Cesar Augusto Suarez Garcia, Anil Adisesh, Christopher J0 Baker. 'University of New Brunswick, Canada

\subsection{6/OEM-2021-EPI.442}

Introduction Occupational encoding is a technique that allows job titles provided by study participants to be categorized according to their role in the labor force. Encoding has primarily been a slow error-prone manual process which is ripe for automation.

Objectives Our goals was to design and test an automated coding prototype using machine learning techniques.

Methods The prototype classification system ENENOC (the ENsemble Encoder for the National Occupational Classification) is comprised of series of steps involving data cleaning, exact match search, multi classifier ensembling, hierarchical classification, and multiple output selection. In the absence of exact matching between job title input and NOC category descriptions, the input data is embedded using the TF-IDF algorithm and Doc2Vec. The embeddings are fed into a hierarchical, ensemble classifier that uses classical machine learning techniques: Random Forests, Support Vector Machine and K-Nearest Neighbour. Ensemble encoding is achieved using a majority-voting system. The hierarchical two tier classification methodology first predicts the first digit of the NOC code followed while the second tier predicts the second third and fourth digit of the NOC code for the input data. The combined approach produces a single, 4-digit code as a top choice, as well as four alternate NOC codes, that serve as additional ranked choice based on the Doc2Vec model.

Results The prototype was benchmarked on a manually annotated data set comprising of 64,000 records. It produced a top-1 Per-Digit Macro F1-Score of 0.65 and a top-5 Per-Digit Macro F1-Score of 0.76 , both of which are highly within published accuracy ranges for manual coding (44\% to $89 \%$ inter-annotator agreement). ENENOC coded 30,000 job titles in 3 hours.

Conclusion The ENENOC prototype is a sophisticated ENsemble Encoder for the National Occupational Classification which has state of the art performance accuracy with significant speed improvements over manual coding. 